\title{
Building Mass Rail Transit in Kuala Lumpur: Is its Good Transport Economics?
}

\author{
Hamid AHA ${ }^{1 *}$, Tabassi AA $^{2}$ and Samsurijan MS ${ }^{3}$ \\ ${ }^{1}$ School of Housing, Building \& Planning, USM, Malaysia \\ ${ }^{2}$ Manchester Metropolitan University, UK \\ ${ }^{3}$ School of Management, USM, Malaysia
}

*Corresponding author: Ahmad Hilmy Abdul Hamid, School of Housing, Building \& Planning, USM, Malaysia.

Received Date: February 07, 2020

Published Date: February 11, 2020

\section{Short Communication}

Kuala Lumpur city is hoping to join the public transport club of elite capital cities. Public transport especially of the rail-based system provide mass transportation for urban dwellers in a city. Most major cities in the world have good LRT or MRT systems in place for their citizens. Kuala Lumpur also wish to join these group of successful cities. In 2010 the federal government of Malaysia declared that the MRT line 1 and 2 will be built to increase public transport use in Kuala Lumpur. In July 2017, the MRT line 1 was completed. This paper reviews whether the MRT system is, after all, a good investment economically from the transport point of view.

\section{The KL MRT System}

The KL MRT (line 1) system is $51 \mathrm{~km}$ long starting from Sungai Buloh in the north to Kajang in the south. The system was planned to carry 150000 passengers per day. The tickets are priced from RM1.10 to RM 6.40 for the whole length of the line.

\section{Area of service}

The alignment of the MRT line 1 takes a traveler across Kuala Lumpur city in the north-south direction. The proposed MRT line 2 is supposed to connect Sungai Buloh to Putrajaya, the administrative capital of the country. The MRT line 3 has now been postponed as part of the austerity drive by the new Malaysian government.

\section{Existing Rail, Bus and Taxis Services}

KL already have LRT and monorail lines serving a fair section of the metropolitan area. The Kelana Jaya Line, Sri Petaling Line and Ampang Line are all operated by Rapid Rail. All three lines carry a combined total of 300,000 commuters per day. These lines are supplemented by the KL Rapid bus and city cab and taxi services. Metered taxis are available throughout the city but maybe difficult to get during rush hours. GRAB car e-hailing service now provides a good alternative to taxis and other transport modes.

\section{Cost of Buses \& Operations}

In general, a large size bus carrying some 60 passengers' cost RM500k each. A medium size bus with a capacity of 35-40 will cost RM300k each. A minibus whose capacity is about 20 (including standing passengers) can cost up to RM100k.

Buses use existing roads and each bus type caters for the limitations of the road configurations. Smaller buses are used for narrow winding roads with limited roadside space. The bigger buses are used for wide straight roads with dedicated bus-stops facilities and terminal locations.

If we take the case of the MRT line 1 which cost RM 21 Billion to be built, that same amount could buy 40,000 buses at a cost of RM500k each. If one bus can carry 60 passengers, then 40,000 buses will be able to carry $2.4 \mathrm{M}$ passengers. This number is 16 times more than number of passengers that could ride the MRT line 1 which is 150,000 passengers in a day.

\section{Number of cars removed}

Other criteria used to justify the construction of the MRT system is the number of cars removed from the roadways because of mode shift. Assuming all cars are single occupant vehicles (SOV) then if 150,000 people opted for the MRT then it is argued that the same number of cars will be removed from the city roadways. Using the 
same argument, for buses this would mean some $2.4 \mathrm{M}$ cars would be removed from the roads if people opted for the 40,000 buses provided.

\section{Transport Economics and User Mode Choice}

As indicated earlier, travel mode choice in Kuala Lumpur is quite varied. Besides the private transport such as cars and motorcycles, travelers can choose taxis, buses and rail-based systems. The overall modal split in Kuala Lumpur presently stands at 25\% public transport, still a long way from the targeted $40 \%$ by 2030 declared by the government.

\section{Increasing the reach of bus services}

While the bus service in Kuala Lumpur is as old as the city, its status as popular mode of travel has been a roller coaster. In the late 1970-80s buses were quite popular especially with the presence of mini-buses which were cheap and convenient. System modernization via regularization of service providers saw the many bus operators being replaced by the KL Bus Rapid service. The implementation of the Monorail and LRT lines within the KL city center however somewhat diminished the utilization of buses primarily because bus network generally remained unchanged, catering for existing and historical routes. Many newly developed areas with large number of residents were not privy to bus services.

\section{Conclusion}

The sophisticated and effective transportation infrastructure in cities contributes towards the overall quality of life of urban populations. A good transportation infrastructure is capable of enhancing their accessibility, in which the inhabitants have the opportunity to choose a suitable and comfortable form of transportation. A high level of accessibility affords the urban village populations the ability to adapt with the city surroundings that are ever changing. The transportation infrastructure around a city is essential in assuring the quality of life and well-being of urban village inhabitants. The last decade has witnessed rapid development of rail-based public transportation systems in KL. The opening of the KL Monorail will be a cornerstone in KL's rail infrastructure. Future development will concentrate on expanding the network, adding stations and facilities, improving standards, and improving feeder access. There are many challenges in integrating the diversity of services into a system that satisfies passengers' requirements for efficient, seamless, flexible and inexpensive public transport.

The MRT system while proven elsewhere to be fundamentally useful for reducing private vehicles in cities, has not been able to do the same for Kuala Lumpur. The most dominant drawback of the KL MRT is the ROI per passengers carried. It is shown that the conventional bus system could provide similar carrying capacity at a much lesser cost. Buses can also penetrate a much wider area merely by accessing existing road networks. However, while the MRT system suffers from the first and last mile connectivity issues, bus usage needs to be promoted for it to gain patronage. It is not difficult to envisage that once the bus system has matured and is able to deliver commuters to their destinations, rail-based system such as MRT can then do the heavy haulage of readily available commuters much like the expressway does to carry heavy traffic.

\section{Acknowledgement}

None.

\section{Conflict of Interest}

No conflict of interest. 